\title{
ANÁLISE DE REDES SOCIAIS: AVANÇOS RECENTES E CONTROVERSIAS ATUAIS
}

RESUMO

A análise de redes avançou rapidamente nas últimas três décadas, mas as críticas à abordagem também aumentaram. Este artigo aborda diversas realizações e problemas não resolvidos da abordagem de redes. $\mathrm{N}$ a primeira seção, ilustrarei o valor do modelo de redes em diversas situações empíricas, concentrandome em estudos de centralidade e poder, subgrupos da rede e relações interorganizacionais. Em seguida, discutirei três questões em que a abordagem tem causado controvérsia: a relação entre a análise de redes e a teoria da escolha racional; o papel das normas e da cultura; e a questão da agência humana. Concluirei com alguns exemplos de como os teóricos das redes estão abordando esses problemas.

\section{Mark S. Mizruchi}

University of Michigan

ABSTRACTN etwork analysis has grown rapidly over the past two decades, but criticisms of the approach have increased as well. This article focuses on several accomplishments and unresolved problems of the network approach. In the first section, I illustrate the value of the network model in several substantive areas, focusing on studies of centrality and power, network subgroups, and interorganizational relations. I then discuss three issues over which the approach has provoked controver sy: the relation between network analysis and rational choice theory; the role of norms and culture; and the question of human agency. I conclude with some examples of how network theorists are addressing these problems.

PALAVRAS-CHAVE Redes sociais, análise de redes, relações interorganizacionais, teoria da escolha racional, agência humana. KEYORDS Social networks, network analysis, interorganizational relations, rational choice theory, human agency. 


\section{INTRODUÇÃO}

A análise de redes tem conquistado um número crescente de adeptos nas últimas três décadas. Multiplicam-se as pesquisas que sugerem que as redes sociais influenciam o comportamento de indivíduos e grupos. Com sua crescente popularidade, as críticas à análise de redes também proliferaram. Este artigo atende a dois objetivos. Primeiro, apresentarei um breve panorama da literatura sobre redes em três importantes áreas: centralidade e poder; subgrupos da rede; e relações interorganizacionais. 0 intuito dessa discussão é demonstrar os avanços que têm ocorrido nessas áreas. Segundo, discutirei três questões que têm sido fonte de controvérsias teóricas: a relação entre a análise de redes e a teoria da escolha racional; o papel das normas e da cultura; e a questão da agência humana. Concluirei com uma breve discussão das perspectivas futuras para a análise de redes.

\section{ANTECEDENTES HISTÓRICOS}

A análise de redes tem suas raízes em diversas perspectivas teóricas. Alguns encontram suas origens no trabalho do psiquiatra J. L. Moreno (1934), que desenvolveu uma abordagem conhecida como sociometria, em que as relações interpessoais eram representadas graficamente. Outros as encontram no trabaIho dos antropólogos britânicos J ohn Barnes (1954), Elizabeth Bott (1957) e J. Clyde Mitchell (1969). Ainda outros (Berkowitz, 1982) vêem a análise de redes como um apêndice do estruturalismo francês de Claude Lévi-Strauss (1969).

A análise de redes também pode ser vista como um subtipo do arcabouço geral da sociologia estrutural (Wellman, 1988). A sociologia estrutural é uma abordagem segundo a qual estruturas sociais, restrições e oportunidades são vistas como afetando mais o comportamento humano do que as normas culturais ou outras condições subjetivas. As raízes clássicas da sociologia estrutural são encontradas em Durkheim, Marx e (especialmente) Simmel. A influência deste último sobre a sociologia estrutural decorre de sua preocupação com as propriedades formais da vida social. Para Simmel, certas relações sociais seguiram padrões que assumiram características semel hantes em uma ampla gama de contextos. Em qualquer situação que envolva três agentes, por exemplo, um agente será bem sucedido na medida em que possa explorar um conflito entre os outros dois. Esse padrão pode ocorrer entre pessoas, organizações e até países. Para Simmel, as formas e padrões das relações sociais eram mais importantes do que seu conteúdo. Como colocou Blau (1982, p. 276), os sociólogos estruturalistas estão mais preocupados com a "proporção de agentes isolados num grupo do que com o fato de seus nomes serem Jack e Jim ou Jill e Joan".

Embora possa haver diferenças entre correntes da sociologia estrutural, a maioria dos sociólogos estruturalistas concorda que os fatores objetivos sejam determinantes mais significativos do comportamento do que os subjetivos. A análise de redes é um tipo de sociologia estrutural que se baseia numa noção clara dos efeitos das relações sociais sobre o comportamento individual e grupal.

\section{PRINCÍPIOS E MÉTODOS DA ANÁLISE DE REDES}

O princípio básico da análise de redes é que a estrutura das relações sociais determina o conteúdo dessas relações. Os teóricos das redes rejeitam a noção de que as pessoas são combinações de atributos, ou de que as instituições são entidades estáticas com limites claramente definidos. Os sociólogos usam com freqüência os termos "sociedade", "governo" e "economia", e referemse aos indivíduos usando termos tais como "protestantes de classe média baixa que residem nas áreas urbanas centrais e que votam no Partido Democrata" (White, Boorman e Breiger, 1976, p. 733). Mas esses termos e categorias ofuscam aquilo que, para os teóricos de redes, seria a matéria principal da vida social: as redes concretas de relações sociais, que ao mesmo tempo incorporam e transcendem organizações e instituições convencionais. 0 governo, por exemplo, não é uma instituição fixa e unitária, mas uma série de subunidades, muitas vezes operando em oposição umas às outras, cujos membros desenvolvem coalizões e disputas não apenas dentro das agências e entre elas, mas também com diversos agentes externos ao Estado (Martin, 1991). Seria necessário compreender as relações sociais entre agentes dentro e fora das agências estatais para explicar o desenvolvimento da política governamental, por exemplo.

A discussão de Simmel a respeito de díades e tríades [1917] (1950) ilustra o princípio segundo o qual a estrutura das relações sociais afeta seu conteúdo. Não só a entrada de uma terceira pessoa num encontro entre duas outras altera a natureza da relação entre as duas 
pessoas originais, como, também, a natureza da tríade em si é significativa. Numa tríade fechada, exemplificada no painel $A$ da Figura 1, cada agente interage com os dois outros. Numa tríade hierárquica, como a do painel $B$ da Figura 1, 0 agente central ocupa uma posição de corretagem entre os dois outros, que são obrigados a lidar com o corretor para efetuar comunicação um com o outro. Essas duas estruturas, segundo a teoria das redes, criam formas de interação muito diferentes entre os membros do grupo. 0 potencial de corretagem permite que 0 agente central da tríade extraia benefícios de qualquer situação em que os dois outros agentes procurem se comunicar (Freeman, 1979; Cook, 1982; M arsden, 1982; Gould e Fernandez, 1989).

A análise de redes é, em tese, aplicável a virtualmente qualquer assunto empírico. Embora os analistas de redes tenham abordado uma ampla gama de assuntos, três áreas que mereceram atenção especial por causa de sua relevância teórica são os efeitos da centralidade do agente sobre o comportamento, a identificação de subgrupos da rede e a natureza das relações entre as organizações.

\section{Rede e centralidade do agente}

Durante as décadas de 1950 e 1960, diversos experimentos, a começar pelos realizados no M IT sob a condução de Bavelas (1950; Leavitt, 1951; posteriormente, Hopkins, 1964; Faucheux e Mackenzie, 1966; Mackenzie, 1976), identificaram diferenças consideráveis no caráter das atividades de solução de problemas em grupo entre diversas estruturas de comunicação. De especial importância foi a relação entre a central idade de um agente e sua influência sobre o grupo. Leavitt (1951), por exemplo, demonstrou, por meio de diversos tipos de estrutura de comunicação, que as

Figura 1 - Dois tipos de tríades

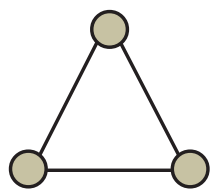

A

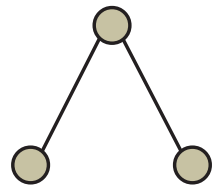

B

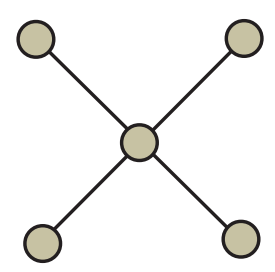

A diferenças de influência entre 0 agente mais central e o menos central aumentavam com a crescente hierarquia das estruturas. A Figura 2 apresenta exemplos de estruturas clássicas, hierárquica e não hierárquica, de cinco agentes. $\mathrm{Na}$ estrutura hierárquica, conhecida como a "roda" (painel A), o agente central controla 0 fluxo de informação entre qual quer par de outros agentes. $\mathrm{N}$ a estrutura não hierárquica ( painel $\mathrm{B}$ ), que neste caso é um "subgráfico máximo completo" em que estão presentes todos os laços possíveis, qual quer membro do grupo pode se comunicar diretamente com qualquer outro. Freeman (1979) desenvolveu uma medida da centralização da rede com base na diferença entre a centralidade da unidade mais central e a das demais unidades. ${ }^{1}$ F reeman demonstra que, por meio do uso dessa medida, a rede hierárquica da Figura 2 apresenta grau de centralização igual a 1 (a maior possível), enquanto a rede não hierárquica da Figura 2 apresenta grau zero (a menor possível).

0 rápido desenvolvimento da análise de redes nos últimos anos levou ao ressurgimento de pesquisas experimentais e não experimentais sobre a relação entre a centralidade e o poder dos agentes sociais. M arsden (1982), Cook et al. (1983), M arkovsky, Willer e Patton (1988), e os artigos da edição especial de setembro/ dezembro de 1992 da publicação Social N etworks fornecem exemplos de trabalhos experimentais e de simulação. Galaskiewicz (1979), Mizruchi (1982), Mintz e Schwartz (1985), e Laumann e Knoke (1987) fornecem exemplos de trabalhos não experimentais que operam no nível interorganizacional de análise. Astley e Zajac (1990), Brass e Burkhardt (1992) e Krackhardt (1992) apresentam exemplos do papel da centralidade nas organizações. Scott (1991), Cook e W hitmeyer (1992), e Mizruchi e Galaskiewicz (1993) oferecem revisões dessa literatura.

Figura 2 - Estruturas hierárquica e não hierárquica

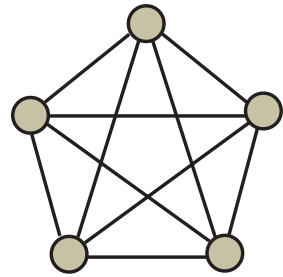

B 
Embora diversos desses estudos tenham demonstrado uma associação positiva entre a centralidade e 0 poder, a associação entre os dois é mais complexa do que sugerem os primeiros estudos. Simulações e resultados experimentais de Marsden (1982, 1987), Cook et al. (1983) e Markovsky et al. (1988) revelaram que, em determinados tipos de estruturas (como a estrutura de acesso restrito apresentada na Figura 3), agentes com elevada centralidade "local" (Nieminen, 1974), tais como 7, 8 e 9, podem ser mais poderosos do que agentes com el evada centralidade "global", como 0 agente $10 .{ }^{2} \mathrm{Em}$ algumas situações, a elevada centralidade pode até representar um empeciIho. Num estudo a respeito da conspiração para a fixação de preços da indústria de equipamentos el étricos dos Estados Unidos ocorrida no início da década de 1960, Baker e Faulkner (1993) desobriram que os agentes centrais foram aqueles com maior probabilidade de serem considerados culpados de crimes, presumivelmente porque suas posições centrais nas redes de comunicação os deixaram mais vulneráveis à detecção. Bonacich (1987) observou que o poder de um agente pode ser maior se suas ligações se derem com agentes relativamente periféricos, que são forçados a lidar com 0 agente focal. $\mathrm{N}$ a maioria das medidas de central idade, contudo, os agentes ligados a agentes periféricos serão menos centrais do que aqueles ligados a agentes centrais. Isso pode explicar algumas situações em que a centralidade e o poder não estão altamente correlacionados. Estudos de Cook et al. (1983) e Marsden (1987) sugerem que a relação centralidade-poder é afetada por serem as redes "positiva" ou "negativamente" conectadas; em redes negativamente conectadas, um laço entre os agentes $A$ e $B$ impede qualquer laço entre $\mathrm{A}$ e $\mathrm{C}$. I sso corresponde à situação relatada por Bonacich, e foi nas redes negativamente conectadas que Cook et al. fracassaram em produzir a associação esperada entre centralidade e poder. $\mathrm{N}$ as redes de Cook et al., os agentes de elevada centralidade local eram mais poderosos do que aqueles com elevados níveis de centralidade global. Marsden (1987) demonstra que o poder relativo dos agentes com elevada centralidade global depende da medida em que os agentes centrais se revel am capazes de formar coalizões.

Apesar da variedade de conclusões sobre a relação entre centralidade e poder, a maioria dos estudos revel ou pelo menos al guma associação substancialmente significativa. Eles condizem, portanto, com um princípio básico da teoria das redes: de que a posição de um agente numa estrutura social tem impacto significativo sobre seu comportamento e bem-estar.

\section{Subgrupos da rede}

O utra área importante da análise de redes é a identificação de subgrupos da rede. A maioria das análises operou dentro de duas grandes tradições, que Burt (1982) intitulou "relacional" e "posicional". Os modelos relacionais se baseiam primordialmente nas técnicas gráfico-teóricas (Harary, N orman e Cartwright, 1965). Seu foco se dá na identificação de "cliques", regiões densamente conectadas das redes em que a totalidade ou maioria dos agentes está diretamente ligada entre si, como na estrutura não hierárquica da Figura 2 (Alba, 1973). Os modelos posicionais se baseiam predominantemente em técnicas de matriz algébrica (Lorrain e White, 1971). Seu foco é na identificação de agentes estruturalmente equival entes, pares de agentes ligados aos mesmos terceiros. A mais proeminente dessas técnicas é o blockmodeling, desenvolvida por Harrison White e seus alunos (White, Boorman e Breiger, 1976; veja na edição especial de junho de 1992 da publicação Social N etworks, um panorama dos avanços recentes). Blockmodels são representações binárias de matrizes relacionais entre agentes de uma rede, permutadas de tal maneira que agentes estruturalmente equival entes se agrupem em submatrizes quadradas, ou "blocos". Os blocos são identificados alternativamente como "blocos-zero" ou "blocos-um", dependendo da densidade dos laços entre os

Figura 3-Rede de acesso restrito com dez agentes

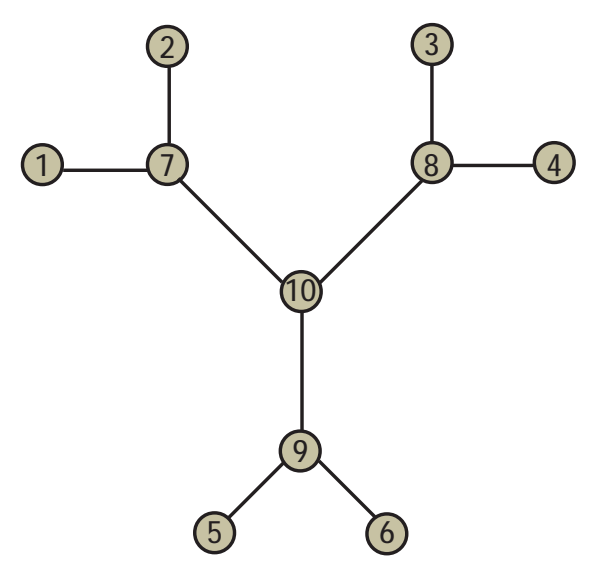


agentes que os compõem. Na prática, usa-se uma determinada densidade como ponto de corte para distinguir blocos-zero de blocos-um. Por exemplo, no blockmodel de uma rede de pesquisa biomédica produzido por White et al., os autores descobriram que densidades de corte na faixa entre 0,10 e 0,50 produziam resultados semelhantes. As representações das estruturas podem ser ainda mais reduzidas por meio do agrupamento de blocos estrutural mente equivalentes em matrizes $2 \times 2$. Os padrões de blocos identificam diferentes tipos de estruturas sociais (W hite et al., 1976). Consideremos, por exemplo, um modelo em que todos os dados brutos sejam escol has de amizade, de tal maneira que os agentes possam indicar outras pessoas específicas como amigas, mas os laços podem ou não ser recíprocos. Se a matriz, quando reduzida a um blockmodel de $2 \times 2$, contiver um blocozero na segunda coluna da primeira linha e blocos-um nas demais células, como na representação a seguir,

\section{0}

11

representaria uma estrutura hierárquica em que os laços vão dos agentes de menor status aos de maior status, mas não o contrário (W hite et al., 1976, p. 742). N esse exemplo, os agentes de status el evado (linha 1) escol hem outros agentes de status el evado (coluna 1), mas não escolhem agentes de baixo status (coluna 2). Os agentes de baixo status (linha 2), por outro lado, escolhem agentes tanto de baixo quanto de al to status (colunas 1 e 2 , respectivamente). Os blockmodels não são as únicas técnicas que empregam a equivalência estrutural como base para o agrupamento. Outras técnicas largamente utilizadas, como a análise fatorial (Allen, 1978), as escalas multidimensionais (Levine, 1972; Laumann e Pappi, 1976) e as técnicas de agrupamento por equival ência estrutural não discreta empregadas por Burt (1982) muitas vezes rendem agrupamentos semelhantes aos produzidos pelos modelos em bloco (veja, em Breiger, Boorman e Arabie, 1975, u ma comparação dos blockmodels com as escalas multidimensionais).

Os proponentes de virtualmente todas as técnicas de agrupamento concordam que os membros de "cliques" ou agrupamentos específicos devem apresentar atitudes e comportamento semel hantes. Mas como os "cliques" gráfico-teóricos se baseiam em laços diretos entre os agentes, ao passo que os blocos e outros agrupamentos posicionais se baseiam em equivalên- cia estrutural, os dois model os levam a previsões diferentes quanto às fontes da influência e da similaridade interpessoal. As relações nos "cliques" se baseiam em laços coesos entre agentes. Nos modelos de coesão, que são os mais largamente utilizados pelos analistas de redes, aquel es que interagem diretamente tenderão a influenciar uns aos outros. Os model os de equivalência estrutural levam a duas interpretações possíveis. U ma, apresentada por F riedkin (1984) e Mizruchi (1993), sugere que agentes estrutural mente equival entes têm a probabilidade de apresentar comportamentos semel hantes porque estão suj eitos a fontes comuns de influência direta. U m argumento al ternativo apresentado por Burt (1987) sugere que os agentes estruturalmente equivalentes, por ocuparem as mesmas posições nas estruturas sociais, competem pelos favores dos ocupantes de outras posições. Por causa dessa competição, os agentes ten dem a imitar os atos de seus pares estruturalmente equivalentes.

Os modelos de coesão e de equival ência estrutural obtiveram considerável apoio na literatura. Alguns dos muitos exemplos que apóiam o modelo de coesão são os estudos de Moore (1979), Friedkin (1984), e Laudmann e Knoke (1987). Os estudos que apóiam o model o de equivalência estrutural incluem os de Burt (1987), Johnson (1986), e Galaskiewicz e Burt (1991). Alguns estudos fornecem apoio às duas abordagens (Mizruchi, 1992, 1993).

\section{Análise de redes e relações interorganizacionais}

Por muito tempo, uma crítica freqüente à análise de rede era de que seus proponentes tiveram sucesso na criação de descrições matemáticas el egantes das estruturas sociais, mas não foram tão bem sucedidos na demonstração de que tais estruturas tenham, realmente, conseqüências comportamentais. Em área al guma essas críticas foram tão enfáticas quanto na do estudo das relações interorganizacionais.

Embora alguns dos primeiros estudos tenham demonstrado que a centralidade nas redes interorganizacionais estaria associada a resultados organizacionais identificáveis, inclusive a probabilidade de sucesso político de uma organização (Galaskiewicz, 1979) e suas estratégias de investimento (Ratcliff, 1980), tais demonstrações foram pouco numerosas até meados da década de 1980. Desde então, contudo, proliferaram estudos que sugerem que a posição de uma empresa nas redes interorganizacionais afeta seu comportamento. Grande parte desses trabalhos surgiu em publicações de Administração de Empresas e se dedicaram aos 
efeitos da composição dos conselhos de administração sobre as estratégias administrativas. Os conselhos de administração são importantes porque os consel heiros de uma empresa muitas vezes são membros dos consel hos de outras, criando aquilo que se chama "diretorias interligadas" [interlocking directorates]. As redes interligadas são a forma mais estudada de relação interorganizacional. Embora haja muitas visões concorrentes a respeito do papel representado por essas interligações, inúmeros teóricos acreditam que elas forneçam um indicador de relações sociais interorganizacionais que, se as previsões da teoria das redes estiverem corretas, deve influenciar o comportamento das empresas (veja revisões desses modelos em Pettigrew, 1992; Mizruchi, 1996).

Diversos estudos sobre a composição de conselhos de administração encontrados na literatura de Administração de Empresas se concentram no papel dos consel heiros externos. Como os consel heiros externos costumam estar associados a outras empresas, essa variável também serve como indicadora da medida em que uma empresa está integrada a redes interorganizacionais. A literatura organizacional sugere que esses laços têm impacto significativo sobre as estratégias corporativas. Num estudo do uso de "greenmail", como é chamada a recompra privada de ações de uma empresa, Kosnik (1987) descobriu que as empresas que resistiam ao greenmail tinham mais consel heiros externos e mais consel heiros representantes de empresas com as quais a empresa focal mantinha transações do que as empresas que eram vítimas do greenmail. Estudos de Cochran, Wood e Jones (1985), Singh e Harianto (1989) e Wade, O'Reilly e Chandratat (1990) constataram que a proporção de membros externos no conselho de administração de uma empresa estava positivamente associado à existência de políticas de "golden parachute" para os altos executivos da empresa. ${ }^{3}$ Davis (1991), num estudo da adoção por empresas de defesas contra a tomada de controle (conhecidas como "poison pills"), descobriu que havia maior chance de uma empresa adotar poison pills se compartilhasse consel heiros com empresas que já as tivessem adotado. Baysinger, Kosnik e Turk (1991) identificaram uma associação negativa entre a proporção de consel heiros externos e as despesas de pesquisa e desenvolvimento das empresas. Clawson e N eustadtl (1989) e Mizruchi (1992) descobriram que as interligações de conselheiros influenciavam as estratégias políticas corporativas. Haunschild (1993), Fligstein e Markowitz (1993), e Palmer et al. (1995) encontra- ram uma associação entre as interligações e a participação das empresas em fusões e aquisições. Boeker e Goodstein (1993) descobriram que empresas com el evada proporção de pessoas de fora em seus consel hos de administração apresentavam maior probabilidade de indicar CEOs de fora da empresa. E Stearns e Mizruchi (1993; Mizruchi e Stearns, 1994) descobriram que a presença de representantes de instituições financeiras no conselho de administração de uma empresa influenciava o tipo e o montante dos financiamentos por ela usados.

Quais são os processos por meio dos quais se sustenta que essas redes influenciam o comportamento empresarial? Um exemplo pode ser encontrado num estudo sobre a ação política das corporações ( M izruchi, 1992). 0 objeto do estudo era a semelhança do comportamento político entre pares de grandes empresas industriais americanas. Entre as variáveis de rede que se esperava levar à semelhança de comportamento, estavam a interdependência econômica das duas empresas, o fato de estar ou não o capital das empresas em poder dos mesmos investidores institucionais, e dois tipos de interligação de conselheiros: a criada por laços diretos entre as empresas e aquela em que ambas as empresas compartil ham consel heiros com uma mesma terceira. Voltando a atenção para os efeitos da interligação, o argumento prosseguia da seguinte maneira: as empresas se envolvem numa situação política com um conjunto de preferências que são al gumas claras, outras incertas. Todos os demais fatores permanecendo constantes, os líderes das empresas interligadas terão maior probabilidade de se comunicar uns com os outros do que os das empresas não interligadas. Entre as idéias que podem ser comunicadas estão informações sobre candidatos políticos. A exposição a informações positivas ou negativas vindas de representantes de outras empresas a respeito de candidatos pode afetar as tomadas de decisão dessa empresa. Quando duas empresas compartilham interligações com diversas outras (laços indiretos, que interpretei como indicador de equival ência estrutural), estão expostas a diversas fontes comuns de informação. I sso aumenta ainda mais a probabilidade de que contribuam para os mesmos candidatos. Os achados desse estudo indicam que a presença de laços indiretos entre empresas estava mais fortemente associada a contribuições aos mesmos candidatos do que a presença de laços diretos. A exposição simultânea a diversas fontes de informação em comum pode ser uma explicação para esse fato.

Até mesmo atividades econômicas cotidianas podem 
ser afetadas pelas relações interorganizacionais. Uzzi (1996), por exemplo, num estudo do setor de vestuário de Nova York, descobriu que empresas em vias de deslocar suas instalações para o exterior avisaram seus fornecedores com meses de antecedência, apesar do fato de que tal conhecimento criaria um incentivo para que os fornecedores recusassem pedidos. Uzzi atribuiu esse comportamento aparentemente irracional às íntimas relações sociais entre os membros do setor.

Granovetter (1985) forneceu um model o teórico genérico para explicar esse comportamento. Desvinculandose da versão de Oliver Williamson (1975) da economia dos custos de transação, Granovetter argumenta que as relações sociais que se desenvolvem entre clientes e fornecedores muitas vezes atenuam, ou mesmo anulam, 0 oportunismo, que segundo Williamson caracteriza as transações no mercado. Williamson (1991) é capaz de explicar o comportamento não oportunista por meio do conceito da especificidade de ativos, em que transações repetitivas criam incentivos para a manutenção das relações, apesar das menores oportunidades de comportamento oportunista. Mas Williamson considera essas situações como aberrações e não como componentes básicos de seu modelo.

\section{SOCIOLOGIA ESTRUTURAL E A TEORIA DA ESCOLHA RACIONAL}

O contraste entre os modelos de Granovetter eWilliamson das transações entre empresas levanta a questão da relação entre os modelos de rede e os modelos econômicos em geral. A sociologia estrutural se desenvolveu nos Estados Unidos durante a década de 1970 como alternativa ao model o normativo que dominara o campo durante as décadas de 1950 e 1960. Esse modelo, mais bem caracterizado pelo trabalho de Tal cott Parsons (1951), sugeria que a base da ordem social estava em crenças generalizadas compartilhadas (valores) e em expectativas de comportamento (normas). Tais valores e normas, segundo o modelo, eram interiorizados predominantemente por meio da socialização na infância. Na medida em que essa socialização fosse bem sucedida, a ação humana prosseguiria voluntariamente de acordo com os valores e normas sociais. ${ }^{4} \mathrm{~A}$ sociologia estrutural, com sua ênfase sobre as restrições e oportunidades que influenciam 0 comportamento, tende a reduzir a importância ou desconsiderar totalmente o papel das normas interiorizadas. As pessoas podem se comportar de acordo com as normas não por as terem interiorizado, mas porque temem as sanções a que poderiam estar sujeitas se as infringissem.

Como a sociologia estrutural e a análise de redes podem ser vistas como alternativas à sociologia normativa, seria útil considerar a relação entre a sociologia estrutural e uma alternativa bastante usada à sociologia normativa: a teoria da escolha racional. A crítica da escolha racional à perspectiva normativa compartilha muitas características com a crítica estruturalista. Os teóricos da escolha racional também se preocupam com a dificuldade em distinguir entre a interiorização da norma e o medo da sanção. Quando observamos os clientes de uma loja pagando por suas compras, abstendo-se do furto, não temos meios para saber se o fazem porque interiorizaram a norma de que furtar é errado ou porque apenas temem as conseqüências caso sejam flagrados. Como é impossível distinguir empiricamente entre as duas hipóteses, e como até mesmo os teóricos normativistas reconhecem que a segunda hipótese também ocorre, os teóricos da escolha racional tendem a supor que é o medo da sanção, não a interiorização da norma, que move o comportamento normativamente prescrito. Os teóricos da escolha racional, portanto, concordam com os teóricos estruturalistas em que, na falta de evidências claras de que os agentes interiorizam as normas, há pouco benefício analítico em admitir que o façam (Hechter, 1987). Ademais, os dois modelos se preocupam com as oportunidades e restrições com que se deparam os agentes. A sugestão de Hedström (1993, p. 167) de que a teoria da escolha racional "presume que, em geral, as variações do comportamento individual são explicadas por diferenças entre as estruturas de oportunidade com que se deparam os agentes e não por variações da sua composição interna" também poderia se aplicar à sociologia estrutural.

0 ponto de divergência entre a sociologia estrutural e a teoria da escolha racional é a análise que fazem dos determinantes do comportamento. Os teóricos da escolha racional normalmente presumem que os indivíduos adentram situações sociológicas dotados de preferências formadas exogenamente e que se mantêm constantes pela duração do encontro social. Essa premissa permitiu aos teóricos da escolha racional desenvolver modelos convincentes e rigorosos de resultados sociais, mas que muitas vezes são empiricamente suspeitos devido às premissas simplificadoras adotadas em sua geração. A sociologia estrutural não traz em seu bojo premissas inerentes quanto à racionalida- 
de dos agentes humanos. Mas nada há no modelo estrutural que exclua a racionalidade humana, e muitos sociólogos estruturalistas admitem, implícita ou expressamente, um modelo de agente racional (Burt, 1982; Granovetter, 1985; M izruchi, 1992). As principais diferenças entre 0 modelo estrutural e o modelo de escolha racional são que, no primeiro (1), as preferências humanas são consideradas endógenas, ou seja, a formação das preferências é tida como algo a ser explicado, e no segundo (2), a ação humana é tida como sendo afetada por estruturas sociais explicitamente definidas.

Como exemplo das diferenças entre as duas perspectivas, apresentei uma distinção entre o que chamo de interesses "individuais" e "estruturais" (Mizruchi e Potts, 1998; veja também Burt, 1982, capítulo 5). Como os teóricos das redes argumentam que todos os interesses são endógenos, uso como principal ferramenta analítica o conceito de interesses individuais. Um interesse individual é uma preferência de um agente na ausência de restrições externas. Um interesse estrutural é uma preferência de um agente sujeito a restrições sociais, que pode diferir do que seria essa preferência se ausentes tais restrições. Uma empresa que mude seu posicionamento em relação a uma questão política para agradar um cliente poderoso revela um interesse estrutural. Nada há nesse modelo que impeça a empresa de ser vista como um agente racional. M as a ação racional da empresa é considerada dentro de um sistema de restrições identificado pelas estruturas sociais em que a empresa está imersa.

Consideremos um caso retirado de um estudo atual sobre tomadas de decisão políticas (Mizruchi e Potts, 1998). Admitamos que um agente adentre uma situação política com uma determinada posição sobre uma questão e um determinado nível de saliência, este último determinado pela importância da questão para 0 agente. Admitamos também que o agente esteja inserido numa rede de relações de dependência em que os outros de quem 0 agente depende mantêm posições políticas opostas. Se 0 agente determinar que a necessidade de manter relacionamentos positivos com os outros de quem depende supera a importância dada à questão, poderá decidir mudar para a posição do outro agente. No estudo citado acima, realizamos uma simulação que ilustra o quanto os resultados políticos podem ser afetados até mesmo por pequenos níveis de dependência na rede. Embora o modelo contenha determinados el ementos decisórios de escol ha racional, a estrutura social altera sistematicamente os resulta- dos políticos. Esses resultados não podem ser compreendidos sem um exame da estrutura. Para exemplos correlatos, veja a modificação realizada por Marsden (1982) do modelo de poder de Coleman (1990), além de Stokman e Van den Bos (1992).

\section{PROBLEMAS DA ANÁLISE DE REDES E DA SOCIOLOGIA ESTRUTURAL}

A sociologia estrutural revitalizou diversas áreas da Sociologia, inclusive a teoria do movimento social, o estudo da desigual dade social, a sociologia do desenvolvimento e até mesmo a pesquisa sobre formação de atitudes. A abordagem estruturalista forçou os pesquisadores a considerar aspectos do mundo social que até então haviam recebido atenção insuficiente, mas um foco exagerado sobre as restrições estruturais faz com que nossas explicações sejam incompletas em duas áreas: as origens e o conteúdo das preferências, e os efeitos da agência humana. Essas áreas tem sido um foco predileto das críticas recentes à análise de redes.

\section{0 papel das normas}

Apesar das críticas anteriormente mencionadas, uma vantagem do modelo normativo é sua tentativa explícita de identificar tanto as origens quanto o conteúdo das visões de mundo das pessoas. A teoria das redes pode explicar por que, dado que os amigos de uma pessoa sejam liberais do ponto de vista político, a pessoa em questão também tende a apresentar posições liberais. Como observamos na seção anterior, tal endogeneidade das preferências é uma vantagem do modelo estrutural em relação ao da escolha racional. $M$ as a abordagem de redes não é capaz de explicar por que determinados grupos de pessoas mantêm posições liberais. Suponhamos que um agente seja membro da classe operária de uma nação industrializada. Historicamente, os membros da classe operária tendem a apoiar os partidos trabal histas ou socialistas. Nem sempre é possível determinar se uma pessoa apóia candidatos de orientação trabalhista porque estes atendem a seus interesses individuais (ou, em outras pal avras, que esse apoio existiria mesmo na ausência de influência de outros), ou se a pessoa apóia esses candidatos por causa da influência de outros. Algumas versões do modelo estruturalista podem explicar por que alguém teria interesse em votar em candidatos trabal histas ou socialistas mesmo que não estivesse sob a influência de outros. N esse caso, cada pessoa vota de acordo com os 
próprios interesses econômicos. Mas o modelo de rede não explica por que al guém vota em determinado candidato sem ser influenciado por outros, pois não é capaz de determinar o conteúdo das preferências de uma pessoa. [ Até a premissa de que seja possível identificar objetivamente os interesses econômicos de um eleitor é em si problemática. Seria possível argumentar, por exemplo, que, a longo prazo, medidas protecionistas prejudicariam a economia, o que poderia prejudicar os trabal hadores que as medidas pretendiam proteger. Isso poderia explicar por que, nos Estados U nidos, uma parcela significativa dos trabal hadores apóia candidatos políticos conservadores].

É possível argumentar que as preferências de todas as pessoas, independentemente do quão isoladas possam parecer, foram influenciadas por outras em al gum ponto. Mas se uma preferência é resultado de uma experiência de socialização anterior, então o modelo estrutural perde grande parte de sua vantagem analítica sobre o normativo, já que a preferência da pessoa é conduzida por normas interiorizadas e não por relações sociais que estão ocorrendo. No exemplo anterior, 0 apoio do trabalhador ao Partido Trabal hista poderia ter se cristalizado durante a social ização infantil. Ademais, muitos argumentos estrutural istas somente são viáveis por causa de uma presunção implícita de normas bem estabelecidas, embora não seja necessário que os agentes interiorizem essas normas, desde que concordem em respeitá-las (Sciulli, 1992). 0 estudo de Baker (1984) sobre a volatilidade de preços no pregão da Bolsa de Chicago é um bom exemplo. Baker concluiu que os preços flutuavam mais nos grandes grupos do que nos de menor porte porque a interação face a face entre os corretores era mais difícil nos grupos maiores. Mas o estudo de Baker só funciona se admitirmos que os participantes da Bolsa concordem com as regras do pregão.

Se for possível identificar as preferências políticas de um agente, e se essas preferências prevêem o comportamento mesmo na ausência de influência interpessoal clara, então os atributos pessoais de um agente, como classe ou raça, são capazes de gerar previsões precisas. Assim, podemos prever que um membro da classe operária irá apoiar o Partido Trabalhista simplesmente pela observação de que a pessoa pertence à classe operária. N esse caso, um modelo estrutural pode ser de utilidade, mas um modelo de rede em que o uso de categorias é desprezado pode ser desnecessário. Mesmo que o pensamento por categoria muitas vezes ofusque os processos sociais por meio dos quais as preferências se formam e a ação ocorre, ele também pode fornecer um poder preditivo que, em alguns casos, supera o de um modelo de rede.

Esse argumento representa a base de uma crítica da análise de redes formulada por Brint (1992). Concentrando-se especificamente no trabal ho de W hite, Brint sugere que as categorias são freqüentemente as bases das identidades e papéis sociais que contêm os vários preceitos normativos que fornecem as bases para ação. Em sua réplica, White (1992a) não nega que as normas sejam necessárias para que as estruturas sociais operem, mas argumenta que as estruturas sociais são uma pré-condição necessária para a geração de arcabouços normativos comuns. Um dos motivos pelos quais pessoas de diferentes grupos numa dada sociedade (jovens urbanos versus suburbanos de meiaidade, por exemplo) têm dificuldades de comunicação é o fato de que seu isolamento social Ihes proporcionou poucas oportunidades de experiências e entendimentos comuns.

Com efeito, o conceito de equival ência estrutural foi inicialmente desenvolvido para captar o conceito de papel. M ais tarde se descobriu que a representação matemática da equival ência estrutural era incapaz disso porque a equivalência estrutural era definida em termos dos laços com os mesmos agentes específicos (de modo que dois pais só poderiam ser estruturalmente equival entes se tivessem os mesmo filhos; ver Winship [1973] 1988). Os teóricos de redes desenvolveram o conceito de equivalência de papéis para lidar com essa questão. Agentes de papéis equival entes não precisam ter laços com o mesmo agente, mas apenas estar envolvidos em relacionamentos de tipo semel hante com agentes de tipo semel hante (veja, além do artigo de Winship, Sailer, 1978; W hite e Reitz 1983; Burt, 1990; Borgatti e Everett, 1992; Mizruchi, 1993). O conceito de equival ência de papéis e suas variantes, inclusi ve a equival ência automórfica e a regular, reconhecem o fato de que os papéis sociais tendem a vir acompanhados de prescrições normativas. 0 que importa é que esses papéis sejam definidos em termos da posição que uma pessoa ocupa na estrutura social.

É improvável que o debate entre os teóricos das redes e os proponentes da posição de Brint se resolva logo, mas o volume de diálogos aumentou significativamente nos últimos anos. Aqueles que enfatizam o papel da cultura muitas vezes reconhecem a importância da estrutura social. E os analistas de redes reconhecem cada vez mais a existência de lacunas nas explicações estruturais cujo preenchimento pode exigir 
explicações culturais ou normativas. Ainda assim, mesmo que os defensores de uma posição específica admitam que a alternativa tem al go a oferecer, o debate levanta uma questão de estratégia analítica: qual abordagem, qual ponto de partida, fornece maior alavancagem explanatória? Essa pergunta também fica em aberto. 0 que se pode dizer é que, como estratégia de pesquisa, a análise de redes tem demonstrado considerável poder analítico. ${ }^{5}$

\section{0 papel da agência}

Um segundo problema que compartilham a análise de redes e a sociologia estrutural é o tratamento dado à agência humana. [0 modelo normativo muitas vezes não tem melhor sina nesse aspecto, mas isso é irrelevante para os fins deste artigo.] Os teóricos estruturalistas enfatizam o quanto a ação humana é afetada por restrições e oportunidades. Mas não foram capazes de desenvolver um model o abrangente da agência humana.

0 argumento de que os modelos estruturais muitas vezes apresentam concepções pouco desenvolvidas da agência humana foi levantado por diversos teóricos (Giddens, 1984; Haines, 1988; Cohen, 1989; Brint, 1992). Uma primeira tentativa explícita de incluir a agência numa teoria estrutural foi apresentada por Burt (1982). No modelo de Burt, a estrutura social afeta a ação tanto direta quanto indiretamente por meio de seus efeitos sobre os interesses do agente. A ação é vista, portanto, como capaz de modificar a própria estrutura social (Burt, 1982, p. 9). Alguns críticos (Haines, 1988; Cohen, 1989) argumentaram, contudo, que até mesmo Burt fal ha em desenvolver um modelo que explique como exatamente a ação social modifica as estruturas sociais. Haines (1988), partindo de Giddens, deu ênfase à natureza recursiva da ação humana, em que as ações simultaneamente são afetadas pel as estruturas sociais e as recriam. Diversos trabalhos recentes de teóricos das redes nos aproximaram mais de um modelo teoricamente rigoroso da agência. Dois deles em especial, de autoria de Burt (1992) e White (1992b), lidaram explicitamente com essa questão. ${ }^{6}$

Burt se preocupa com a maneira como os agentes identificam e exploram oportunidades nos sistemas sociais, espaços vazios que ele chama de "buracos estruturais". Essa atenção aos espaços vazios nas estruturas sociais foi um componente significativo do trabalho de White (1970, 1992a; White, Boorman e Breiger, 1976). Ao preencher um buraco, um agente aumenta sua probabilidade de mobilidade ascendente, mas também altera a estrutura de tal forma que deixa de existir um buraco na mesma posição. Burt demonstra que os agentes habilidosos no preenchimento de buracos estruturais e na maximização de seus laços sociais (por meio da diminuição do número de contatos redundantes, por exemplo) têm maior mobilidade ascendente numa organização do que os agentes de menor sucesso no uso e na alteração da estrutura social. Mizruchi e Stearns (2001), num estudo com funcionários de bancos comerciais, demonstram que aqueles capazes de conceber redes com contatos não redundantes apresentavam chance desproporcionalmente maior de fechar negócios com seus clientes corporativos.

White (1992b) procurou reconceitualizar a ação humana em termos de identidade em busca de controle. Identidade é qualquer forma de atividade a que possamos atribuir significado. Existe apenas na medida em que os agentes são capazes de se diferenciar uns dos outros. A ação humana é, assim, uma busca constante de nichos nos quais se possam sustentar identidades. Além de buscarem um nicho peculiar, os agentes de White procuram criar ambigüidade perante aqueles com que interagem, ao mesmo tempo em que mantêm a previsibilidade para si mesmos. 0 estudo de caso de Padgett e Ansell (1993) sobre a ascensão ao poder de Còsimo dei Medici na Florença renascentista é um exemplo desse tipo de ação. Còsimo manteve seu sucesso evitando que suas intenções se revelassem, e evitando compromisso com metas específicas, mantendo, assim, suas opções em aberto.

\section{CONCLUSÃO}

A sociologia estrutural e a análise de redes, com a ênfase que dão a forças sociais objetivas e observáveis e à sua capacidade de gerar hipóteses falsificáveis, representam um avanço em relação a alguns dos aspectos problemáticos dos modelos normativos tradicionais. M as os model os estruturais são limitados por sua incapacidade de lidar com o conteúdo cultural da ação social e por sua concepção pouco desenvolvida da agência humana. A discussão acima sugere que a análise de redes é particularmente promissora no que se refere ao tratamento das questões de cultura e agência humana que vêm representando problemas para a sociologia estrutural de modo geral. Com a ênfase que dá às relações sociais concretas em vez de às categorias, a análise de redes apresenta uma concepção mais dinâmica da ação social do que os modelos normativos ou estruturais tradicionais. 
É importante reconhecer que a análise de redes pode tanto complementar quando suplantar as perspectivas existentes. Um bom exemplo de como a análise de redes pode ampliar o poder analítico de uma teoria alternativa é sua relação com aquele que talvez seja o mais popular modelo sociológico das organizações: a teoria institucional. Esta teoria se baseia na premissa de que as organizações, em vez de fornecer soluções racionais para problemas bem definidos, têm igual chance de reforçar os símbolos ou "mitos" sociais (Meyer e Rowan, 1977; Powell e DiMaggio, 1991). Como parte desse reforço, as organizações constantemente buscam legitimidade a partir de seus ambientes externos. Em vez da busca de uma noção objetiva de eficiência, o comportamento organizacional se torna uma busca da legitimidade. Um argumento importante desse model o foi apresentado por DiM aggio e Powell (1983). Esses autores afirmam que as formas organizacionais das sociedades modernas assemelham-se umas às outras não porque sejam necessariamente as mais eficientes, mas porque a necessidade de legitimidade exige isso delas. DiMaggio e Powell identificam três tipos desse "isomorfismo": o coercitivo, em que as organizações desenvolvem estruturas para se adequarem às expectativas de outras organizações das quais são dependentes; o mimético, em que as organizações, sob condições de incerteza, simplesmente adotam as estruturas de seus pares; e o normativo, em que experiências de socialização semelhantes levam a visões de mundo que geram tipos semel hantes de soluções prescritas a problemas organizacionais. 0 que falta ao modelo de DiM aggio e Powell, e a análise de redes é capaz de suprir, é uma concepção detal hada dos processos pelos quais se transmite 0 isomorfismo. 0 isomorfismo coercitivo tende a ocorrer em situações de relacionamento direto e coeso entre organizações. 0 isomorfismo mimético tende a ocorrer quando as organizações observam e tentam acompanhar seus pares em equivalência estrutural ou de papéis. A teoria das redes não é capaz de nos dizer por que há símbolos e mitos específicos que as organizações procuram reforçar. Mas pode explicar por que al gumas formas e não outras são adotadas, independentemente de serem ou não mais eficientes em termos objetivos. N esse sentido, embora a teoria de redes possa exigir uma perspectiva institucional ou outra semelhante para explicar completamente um fenômeno, a teoria institucional também pode precisar da teoria de redes, a qual pode tornar mais concretos e rigorosos os conceitos da primeira.
De fato, a versatilidade é um dos pontos fortes da análise de redes. Não só pode ser usada conjuntamente com a teoria institucional, como também é compatível com ela e é capaz de ampliar os modelos da ecologia populacional e dos custos de transação. A noção de nicho organizacional da ecologia populacional foi reconceitual izada nos termos da equivalência estrutural por DiMaggio (1986; veja também, Burt, 1992). E o conceito de custos de transação foi formalizado em análises de corretagem (Marsden, 1982) e ampliado na discussão de Granovetter (1985) sobre imersão. A ligação entre a anál ise de redes e o modelo de dependência de recursos já está bem estabel ecida e não precisa ser repetida aqui (Burt, 1983; Pfeffer, 1987; Mizruchi, 1992).

Embora a luta dos pesquisadores em análise de redes prossiga contra os problemas teóricos acima discutidos, há motivos para otimismo. U ma atenção maior dada à questão da agência gerou avanços significativos nos últimos anos. À medida que os teóricos aproveitam as premissas estocásticas que há por trás dos modelos de rede, avanços em relação ao dilema agência-estrutura parecem iminentes. Maiores esforços para reconhecer a importância da cultura também têm sido alentadores [veja, por exemplo, o trabalho de White (1993) sobre arte, e trabalhos sobre redes e cultura na revista Poetics]. Como demonstrou White, a análise de redes pode ser de grande valia no exame dos contextos por onde a cultura se transmite.

A análise de redes é uma das abordagens que mais crescem no âmbito das Ciências Sociais. Acumulamse evidências de que a ação humana é afetada pelas relações sociais em que os agentes estão imersos. Apresentei, aqui, apenas uma pequena amostra dessas evidências. $O$ bolo vai continuar crescendo.

\section{AGRADECIMENTOS}

Esta pesquisa recebeu o apoio da U.S. N ational Science Foundation (bolsa \#SBR-9308443). 0 autor agradece aJulia Adams, Peter Hedström, Richard Swedberg e a três revisores anônimos por seus comentários sobre versões anteriores.

\section{NOTAS}

\footnotetext{
${ }^{1}$ Essa medida apresenta três variantes, com base nas três concepções de centralidade de Freeman abaixo discutidas. 0 cálculo básico envolve a soma das diferenças entre a centralidade da unidade mais central e de todas as demais unidades e a divisão dessa soma pela máxima soma possível das diferenças numa rede do porte em questão.
} 
${ }^{2}$ Embora possa ter havido diversos avanços nos últimos anos, o argumento teórico mais abrangente sobre a centralidade ainda está no trabalho de Freeman (1979). Freeman distinguiu três tipos de centralidade, baseados em grau [degree], proximidade [closeness] e intermediação [betweenness]. 0 grau se refere ao número de laços diretos entre um e outros agentes de uma rede. A proximidade se refere à medida em que um agente pode alcançar um grande número de outros, com pequeno número de passos. $\mathrm{E}$ a intermediação se refere à medida em que um agente cria um caminho singular até outros agentes, de modo que estes precisem passar por ele se quiserem se comunicar. A definição de centralidade apresentada por Bonacich (1972 e elaborada e modificada em 1978) também foi muito influente. Veja, ainda, Mizruchi et al. (1986), Stephenson e Zelen (1989), e Friedkin (1991).

${ }^{3}$ Os acordos de golden parachute são lucrativos pacotes demissionários garantidos aos principais executivos em caso de demissão involuntária. Esses planos proliferaram no mundo empresarial dos Estados U nidos durante a década de 1980 .

${ }^{4}$ Evidentemente o modelo era bem mais complexo e variado do que sugere essa breve descrição. Mas dava grande ênfase ao papel das normas aprendidas na influência sobre o comportamento.

${ }^{5}$ É necessário considerar o papel da cultura para entender o conteúdo das prescrições normativas associadas às relações sociais. Uma concepção de cultura que poderia ser útil à sociologia estrutural foi apresentada por Swidler (1986). Ele argumenta que se pode compreender melhor a cultura como um sistema de comportamentos aprendidos que os agentes usam para negociar suas atividades diárias. Esses comportamentos são aprendidos em contextos sociais específicos e precisam ser continuamente reforçados nesses contextos. 0 que esse modelo tem de valioso éa visão de que as normas não são necessariamente parte de um sistema generalizado de valores, mas, ao contrário, desenvolvem-se em contextos comportamentais específicos.

${ }^{6}$ Veja também os artigos em Weesie e Flap (1990), que incluem discussões de métodos para lidar com mudanças havidas nas redes ao longo do tempo.

\section{NOTA DA REDAÇÃO}

Este artigo é uma versão gentilmente atualizada pelo autor para a publicação no Fórum de Redes Sociais e Interorganizacionais da RAE.

Artigo originalmente publicado com o título "Social network analysis: recent achievements and current controversies", de Mark S. Mizruchi, na Acta Sociologica, v. 37, n. 4, p. 329-343, 1994. (c) Scandinavian Sociological Association, 1994. Todos os direitos são reservados. Publicado com autorização da Sage Publication Ltd. www.sagepub.co.uk

\section{REFERÊNCIAS BIBLIOGRÁFICAS}

ALBA, R. D. A graph-theoretic definition of a sociometric clique. Journal of Mathematical Sociology, v. 3, n. 1, p. 113-126, 1973.

ALLEN, M. P. Economic interest groups and the corporate elite structure. Social Science Quarterly, v. 58, p. 597-615, 1978.

ASTELY, W. G.; ZAJAC, E. J. Beyond dyadic interdependence. Organization Studies, v. 11, n. 4, p. 481-501, 1990.

BAKER, W. E. The social structure of a national securities market. American Journal of Sociology, v. 89, n. 4, p. 775-811, 1984.

BAKER, W. E.; FAULKNER, R. R. The social organization of conspiracy: illegal networks in the heavy electrical equipment industry. American Sociology Review, v. 58, n. 6, p. 837-860, 1993.

BARNES, J. A. Class and committees in a N orwegian island parish. Human Relations, v. 7, n. 1, p. 39-58. 1954

BAVELAS, A. Communication patterns in task-oriented groups. Journal of the Acoustical Society if America, v. 57, p. 271-282, 1950.

BAYSINGER, B. D.; KOSNIK R. D.; TURK, T. A. Effects of board and ownership structure on corporate $R \& D$ strategy. Academy of $M$ anagement Journal, v. 34, n. 1, p. 205-214, 1991.

BERKOWITZ, S. D. An Introduction to Structural Analysis. Toronto: Butterworths, 1982.

BLAU, P. M. Structural sociology and network analysis: an overview. In: MARSDEN, P. V.; LIN, N. (Eds.). Social Structure and Network Analysis. Beverly Hills, CA: Sage, 1982.

BOEKER, W.; GOODSTEIN, J. Performance and successor of choice: the moderating effects of governance and ownership. Academy of M anagement Journal, v. 36, n. 1, p. 172-186, 1993.

BONACICH, P. Technique for analyzing overlapping memberships. In: COSTNER, H. (Ed.). Sociological M ethodology. San Francisco: Jossey-Bass, 1972.

$\mathrm{BONACICH}, \mathrm{P}$. Power and centrality: a family of measures. American Journal of Sociology, v. 92, n. 5, p. 1170-1182, 1987.

BORGATTI, S. P.; EVERETT, M. G. The notion of position in social network analysis. In: MARSDEN, P. V. (Ed.). Sociological Methodology. Oxford: Basil Blackwell, 1992.

BOTT, E. Family and Social N etwork: Roles, N orms, and External Relationships in Ordinary U rban Families. London: Tavistock, 1957.

BRASS, D. J.; BURKHARDT, M. E. Centrality and power in organizations. In: NOHRIA, N.; ECCLES, R. G. (Eds.). N etwork and Organizations. Boston: Harvard Business School Press, 1992. 
BREIGER, R. L.; BOORMAN, S. A.; ARABIE, P. An algorithm for clustering relational data with applications to social network analysis and comparison with multidimensional scaling. Journal of M athematical Psychology, v. 12, n. 3, p. 328-383. 1975.

BRINT, S. Hidden meanings: cultural content and context in Harrison White's structural sociology. Sociological Theory, v. 10, p. 194-208, 1992.

BURT, R. S. Toward a Structural Theory of Action: N etwork M odels of Social Structure, Perception, and Action. New York: Academic Press, 1982.

BURT, R. S. Corporate Profits and Cooptation. New York: Academic Press, 1983.

BURT, R. S. Social contagion and innovation: cohesion versus structural equivalence. American Journal of Sociology, v. 92, n. 6, p. 1297-1335, 1987.

BURT, R. S. Detecting role equivalence. Social Networks, v. 12, p. 83-97, 1990.

BURT, R. S. Structural Holes: The Social Structure of Competition. Cambridge, MA: Harvard University Press, 1992.

CLAWSON, D.; NEUSTADTL, A. Interlocks, PACs, and corporate conservativism. American Journal of Sociology, v. 94, p. 749-773, 1989.

COCHRAN, P. L.; WOOD, R. A.; JONES, T. B. The composition of boards of directors and incidence of golden parachutes. Academy of $M$ anagement Journal, v. 28, n. 3, p. 664-671. 1985.

COHEN, J. S. Structuration Theory. London: Macmillan, 1989.

COLEMAN, J. S. Foundations of Social Theory. Cambridge: Harvard University Press, 1990.

COOK, K. S. Network structures from an exchange perspective. In: MARSDEN, P. V.; LIN, N. (Eds.). Social Structure and Network Analysis. Beverly Hills, CA: Sage, 1982.

COOK, K. S.; EMERSON, R. M.; GILLMORE M. R; YAMAGISHI, T. The Distribution of power in n-person exchange networks: theory and experimental results. American Journal of Sociology, v. 89, p. 275-305, 1983.

COOK, K. S.; WHITMEYER, J. M. Two approaches to social structure: exchange theory and network analysis. Annual Review of Sociology, v. 18, p. 109-127, 1992.

DAVIS, G. F. Agents without principles? The spread of the poison pill through the intercorporate world. Administrative Science Quarterly, v. 36, n. 4, p. 583-613, 1991.

DIMAGGIO, P. J. Structural analysis of organizational fields: a blockmodel approach. In: STAW, B. M.; CUMMINGS, L. L. (Eds.). Research in Organizational Behavior. Greenwich, CT: JAI Press, 1986.

DIMAGGIO, P. J.; POWELL, W. W. The iron cage revisited: institutional isomorphism and collective rationality in organizational fields. American Sociological Review, v. 48, n. 2, p. 147-160, 1983.
FAUCHEUX, C.; MACKENZIE, K. D. Task dependency of organizational centrality: its behavioral consequences. Journal of Experimental Social Psychology, v. 2, p. 361-375, 1966.

FLIGSTEIN, N.; MARKOWITZ, L. Financial reorganization of American corporations in the 1980s. In: WILSON, W. J. (Ed.). Sociology and the Public Agenda. Newbury Park, CA: Sage, 1993.

FREEMAN, L. C. Centrality in social networks: I. Conceptual clarification. Social Networks, v. 1, n. 2, p. 215-239, 1979.

FRIEDKIN, N. E. Structural cohesion and equivalence explanations of social homogeneity. Sociological Methods and Research, v. 12, n. 3, p. 235-261, 1984.

FRIEDKIN, N. E. Theoretical foundations for centrality measures. American Journal of Sociology, v. 96, n. 6, p. 1478-1504, 1991.

GALASKIEWICZ, J. Exchange Networks and Community Politics. Beverly Hills: Sage, 1979.

GALASKIEWICZ, J.; BURT, R. S. Inter-organizational contagion and corporate philanthropy. Administrative Science Quarterly, v. 36, n. 1, p. 88105, 1991.

GIDDENS, A. The Constitution of Society: O utlines of a Theory of Structuration. Cambridge, England: Polity Press, 1984.

GOULD, R. V.; FERNANDEZ, R. M. Structures of mediation: a formal approach to borkerage in transaction networks. In: CLOGG, C. C. (Ed.). Sociological Methodology. Washington, DC: American Sociological Association, 1989.

GRAN OVETTER, M. S. Economic action and social structure: the problem of embeddedness. American Journal of Sociology, v. 91, n. 3, p. 481-510, 1985.

HAINES, V. A. Social network analysis, structuration theory, and the holismindividualism debate. Social Networks, v. 10, p. 157-182, 1988.

HARARY, F.; NORMAN, R.; CARTWRIGHT, D. Structural Models: An Introduction to the Theory of Directed Graphs. N ew York: Wiley, 1965.

HAUNSCHILD, P. R. Interorganizational imitation: the impact of interlocks on corporate acquisition activity. Administrative Science Quarterly, v. 38, n. 4, p. 564-592, 1993.

HECHTER, M. Principles of Group Solidarity. Berkeley: University of California Press, 1987.

HEDSTRÖ M, P. Introduction to this special issue on rational choice theory. Acta Sociologica, v. 36, n. 3, p. 167-169, 1993.

HOPKINS, T. K. The Exercise of Influence in Small Groups. Totowa, N]: Bedminster. 1964.

JOHNSON, J. C. Social networks and innovation adoption: a look at Burt's use of structural equivalence. Social Networks, v. 8, p. 343-364. 1986.

KOSNIK, R. D. Greenmail: a study of board performance in corporate governance. AdministrativeScience Quarterly, v. 32, n. 2, p. 163-185. 1987. 


\section{MARK S. MIZRUCHI}

KRACKHARDT, D. The strength of strong ties: the importance of philos in organizations. In: NOHRIA, N.; ECCLES, R. G. (Eds.). N etworks and Organizations. Boston: Harvard Business School Press, 1992.

LAUMANN, E. O.; KNOKE, D. The Organizational State: Social Choice in National Policy Domains. Madison: University of Wisconsin Press, 1987.

LAUMANN, E. O.; PAPPI, F. U. N etworks of Collective Action: A Perspective on Community Influence Systems. N ew York: Academic Press, 1976.

LEAVITT, H. J. Some effects of certain communication patterns on group performance. Journal of Abnormal and Social Psychology, v. 46, n. 1, p. 3850, 1951.

LEFIER, E. M.; WHITE, H. C. A structural approach to markets. In: MIZRUCHI; M. S.; SCHWARTZ, M. (Eds.). Intercorporate Relations. N ew York: Cambridge University Press, 1987

LEVI-STRAUSS, C. Elementary Structures of Kinship. Boston: Beacon, 1969.

LEVINE, J. H. The Sphere of Influence. American Sociological Review, v. 37, p. 14-27, 1972.

LORRAIN, F.; WHITE, H. C. Structural equivalence of individuals in social networks. Journal of Mathematical Sociology, v. 1, p. 49-80, 1971.

MACKENZIE, K. D. A Theory of Group Structures. New York: Gordon and Breach, 1976.

MARKOW SKY, B.; WILLER, D.; PATTON, T. Power relations in exchange networks. American Sociology Review, v. 53, n. 2, p. 220-236, 1988.

MARSDEN, P. V. Brokerage behavior in restricted exchange networks. In: MARSDEN, P. V.; LIN, N. (Eds.). Social Structure and Network Analysis. Beverly Hills, CA: Sage, 1982.

MARSDEN P. V. Elements of interactor dependence. In: COOK, K. S. (Ed.). Social Exchange Theory. Newbury Park, CA: Sage, 1987.

MARTIN, C. J. Shifting the Burden: The Struggle over Growth and Corporate Taxation. Chicago: University of Chicago Press, 1991.

MEYER, J. W.; ROWAN, B. Institutionalized organizations: formal structure as myth and ceremony. American Journal of Sociology, v. 83, n. 2, p. 340363, 1977.

MINTZ, B.; SCHWARTZ, M. The Power Structure of American Business. Chicago: University of Chicago Press, 1985.

MITCHELL, J. C. Social Networks in U rban Situations. Manchester, UK: Manchester University Press, 1969.

MIZRUCHI, M. S. The American Corporate Network: 1904-1974. Beverly Hills, CA: Sage, 1982.

MIZRUCHI, M. S. The Structure of Corporate Political Action: Interfirm Relations and Their Consequences. Cambridge, MA: Harvard University Press, 1992.
MIZRUCHI, M. S. Cohesion, equivalence, and similarity of behavior: a theoretical and empirical assessment. Social Networks, v. 15, p. 275-307, 1993.

MIZRUCHI, M. S. What do interlocks do? an analysis, critique, and assessment of research on interlocking directorates. Annual Review of Sociology, v. 22, p. 271-298, 1996.

MIZRUCHI, M. S.; GALASKIEWICZ, J. Networks of interorganizational relations. Sociological M ethods and Research, v. 22, n. 1, p. 46-70, 1993.

MIZRUCHI, M. S.; MARIOLIS, P.; SCHWARTZ, M.; MINTZ, B. Techniques for disaggregating centrality scores in social networks. In: TUMA, N. B. (Ed.). Sociological Methodology. Washington: American Sociological Association, 1986

MIZRUCHI, M. S.; STEARNS, L. B. A longitudinal study of borrowing by large american corporations. Administrative Science Quarterly, v. 39, n. 1, p. 118-140, 1994.

MIZRUCHI, M. S.; POTTS, B. B. Centrality and power revisited: Actor success in group decision making. Social Networks, v. 20, p. 353-387, 1998.

MIZRUCHI, M. S.; STEARNS, L. B. Getting Deals Done: The Use of Social Networks in Bank Decision-Making. American Sociological Review, v. 66, n. 5, p. 647-671, 2001.

MOORE, G. The structure of a national elite network. American Sociological Review, v. 44, n. 5, p. 673-692, 1979.

NIEMINEN, U. J. On centrality in a graph. Scandinavian Journal of Psychology, v. 15, p. 322-336, 1974.

PADGETT, J. F.; ANSELL, C. K. Robust action and the rise of the medici, 1400-1434. American Journal of Sociology, v. 98, n. 6, p. 1259-1319, 1993.

PALMER, D.; BARBER, B. M.; ZHOU, X.; SOYSAL, Y. The friendly and predatory acquisition of large u.s. corporations in the 1960s: the other contested terrain. American Sociological Review, v. 60, n. 4, p. 469-499, 1995.

PARSO NS, T. The Social System. N ew York: Free Press, 1951.

PETTIGREW, A. M. On studying managerial elites. Strategic M anagement Journal, v. 13, p. 163-182, 1992

PFEFFER, J. A resource dependence perspective on intercorporate relations. In: MIZRUCHI, M. S.; SCHWARTZ, M. (Eds.). Intercorporate Relations. New York: Cambridge University Press, 1987.

POWELL, W. W.; DIMAGGIO, P. J. (Eds.). The New Institutionalism in Organizational Analysis, Chicago: University of Chicago Press, 1991.

RATCLIFF, R. E. Banks and corporate lending: an analysis of the impact of the internal structure of $t$ he capitalist class on the lending behavior of banks. American Sociology Review, v. 45, p. 553-570, 1980. 
SAILER, R. E. Structural equivalence: meaning and definition, computation and application. Social Networks, v. 1, n. 1, p. 73-90, 1978.

SCIULLI, D. Theory of Societal Constitutionalism: Foundations of a N on-M arxist Critical Theory. New York: Cambridge University Press, 1992.

SCOTT, J. Social N etwork Analysis: A Handbook. Newbury Park, CA: Sage Publications, 1991.

SIMMEL, G. The Triad. In: WOLFF, K. H. (Ed.). The Sociology of Georg Simmel. New York: Free Press, 1950.

SINGH, H.; HARIANTO, F. Management-board relations, takeover risk, and the adoption of golden parachutes. Academy of M anagement Journal, $v$. 32, n. 1, p. 7-24, 1989.

STEARNS, L. B.; MIZRUCHI, M. S. Corporate Financing: Economic and Social Aspects. In: SWEDBERG, R. (Ed.). Explorations in Economic Sociology. New York: Russel Sage Foundation, 1993.

STEPHENSON, K.; ZELEN, M. Rethinking centrality: methods and examples. Social Networks, v. 11, n. 1, p. 1-37, 1989.

STOKMAN, F. N.; VAN DEN BOS, J. M. M. A. two-stage model of policymaking with an empirical test in the u.s. energy-policy domain. In: MOORE, G.; WHITT, J. A. (Eds.). Research in Politics and Society. Greenwich, CT: JAI Press, 1992.

SWIDLER, A. Culture in action: symbols and strategies. American Sociological Review, v. 51, n. 2, p. 273-286, 1986.

UZZI, B. The sources and consequences of embeddedness for the economic performance of organizations: The N etwork Effect. American Sociological Review 61: 674-69, 1996.

WADE, J.; O'REILLY, C. A. III; CHANDRATAT, I. Golden parachutes: Ceos and the exercise of social influence. Administrative Science Quarterly, v. 35, n. 4 , p. 587-603, 1990.

\section{Artigo convidado. Aprovado em 27.04.2006.}

\section{Revisão técnica de Maurício Reinert do Nascimento.}

\section{Mark S. Mizruchi}

Professor de Sociologia e Administração de Empresas da University of Michigan.

Interesses de pesquisa nas áreas de Análise de Redes Sociais, Teoria das Organizações, Sociologia

Econômica e Sociologia Política.

E-mail: mizruchi@umich.edu.

Site: বhttp://www-personal.umich.edu/ mizruchi/>

Endereço: Department of Sociology, University of Michigan, Ann Arbor - Michigan - USA, 48109.
WEESIE, J.; FLAP. H. (Eds.). Social N etworks through Time. Utrecht, Netherlands: ISOR, 1990.

WELLMAN, B. Network analysis: from method and metaphor to theory and substance. In: WELLMAN, B.; BERKOWITZ, S. D. (Eds.). Social Structures: A N etwork Approach. New York: Cambridge University Press, 1988.

WHITE, D. R.; REITZ, K. P. Graph and semigroup homomorphisms on networks of relations. Social N etworks, v. 5, p. 193-234, 1983.

WHITE, H. C. Chains of O pportunity. Cambridge, MA: Harvard University Press, 1970.

WHITE, H. C. Varieties of markets. In: WELLMAN, B.; BERKOWITZ, S. D. (Eds.). Social Structures: A N etwork Approach. New York: Cambridge University Press, 1988.

WHITE, H. C. Social grammar for culture: reply to stephen brint. Sociological Theory, v. 10, n. 2, p. 209-213, 1992a.

WHITE, H. C. Identity and Control: A Structural Theory of Social Action. Princeton, NJ: Princeton University Press, 1992b.

WHITE, H. C. Careers and Creativity: Social Forces in the Arts. Boulder, CO: Westview, 1993.

WHITE, H. C.; BOORMAN, S. A.; BREIGER, R. L. Social structure from multiple networks i - blockmodels of roles and positions. American Journal of Sociology, v. 81, n. 4, p. 730-780, 1976.

WILLIAMSON, O. E. Markets and Hierarchies: Analysis and Antitrust Implications. New York: Free Press, 1975.

WILLIAMSON, O. E. Comparative economic organization: the analysis of discrete structural alternatives. Administrative Science Quarterly, v. 36, n. 2, p. 269-296, 1991.

WINSHIP, C. Thoughts about roles and relations: an old document revisited. Social Networks, v. 10, p. 209-231, 1988. 